\title{
Poling of glass modified by femtosecond irradiation
}

\author{
Costantino Corbari, John D. Mills, Peter G. Kazansky \\ Optoelectronics Research Centre, University of Southampton, S017 1BJ. UK \\ Jeremy J. Baumberg \\ Department of Physics and Astronomy, University of Southampton, S017 1BJ. UK
}

\begin{abstract}
Thermal poling of silica glass modified by femtosecond laser irradiation has been demonstrated. Evidence of second-order nonlinearity modulation between the treated and non-treated regions has been observed. The technique was used for $\chi^{(2)}$ grating fabrication.
\end{abstract}

Poled glass is an attractive material for nonlinear optics applications [1]. The low second-order nonlinearity achieved so far represents the main drawback of this technology, but efficient quasi-phase-matching in periodically poled silica fibres would compensate for this limitation [2] and lead to devices such as wavelength converters for wavelength-division-multiplexer re-routing and switching, and frequency doubling in lasers. Various techniques have been used for quasi-phase-matched grating fabrication including periodical electrodes, periodic UV-erasure or erasure by exposure to a femtosecond laser beam [3].

In this work we achieved a $\chi^{(2)}$ modulation in the glass by periodically modifying the silica with femtosecond laser pulses and by subsequent poling [4]. A grating of $20 \mu \mathrm{m}$ period and $4 \mu \mathrm{m}$ line width was written into a silica glass plate (Herasil 1) by scanning with the focused beam produced by an amplified Ti:Sapphire laser operating at $850 \mathrm{~nm}, 150 \mathrm{fs}$ pulse duration and $250 \mathrm{kHz}$ repetition rate. The red luminescence was observed in the irradiated regions indicating that non-bridging oxygen defects were created [5].

Thermal poling was carried out in air for 15 minutes at $280{ }^{\circ} \mathrm{C}$ with $4 \mathrm{kV}$ applied. After poling the grating was investigated using a modelocked and Q-switched $(\lambda=1064 \mathrm{~nm}) \mathrm{Nd}$ :YAG laser, with a focused spot size of $20 \mu \mathrm{m}$. The second harmonic was subsequently imaged on a CCD camera. Translating the grating relative to the incident laser, a $\chi^{(2)}$ modulation was observed across the grating indicating that the $\chi^{(2)}$ is larger in the Ti:Sapphire irradiated regions.

Tests were performed with the Nd:YAG laser both at normal and tilted incidence. In the region well away from the grating, for inclined incidence, second harmonic was produced in a well defined Gaussian beam (Fig.1(a); the brightness is enhanced compared to subsequent images to make the spot visible).
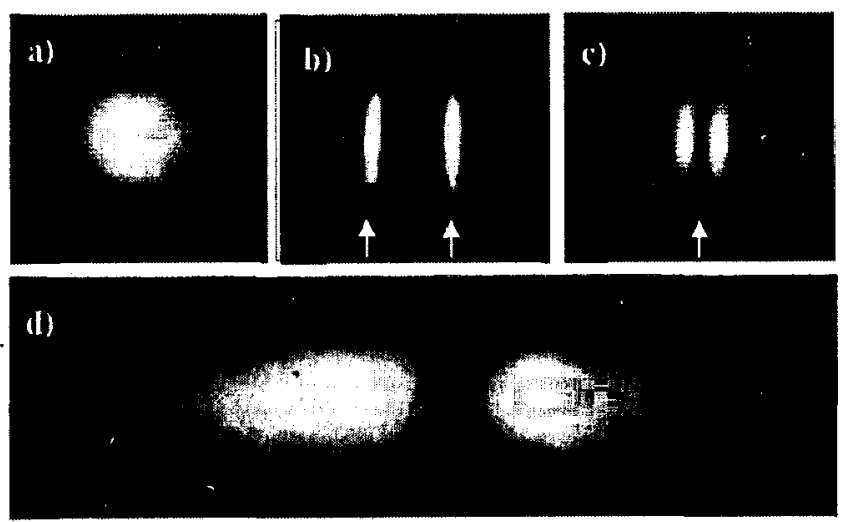

Fig. 1. Imaging of the SHG. (a) Tilted incidence and well away from the grating. The brightness is enhanced compared to subsequent images to make the spot visible. (b) Inclined incidence but on the grating. The SHG from two grating lines (indicated by the arrows) can be seen. (c) Normal incidence. The pump beam is centered on one line: near-field and (d) farfield. 
Also at inclined incidence, in the grating region a well-defined $\chi^{(2)}$ modulation was observed having the same period as the grating. The second harmonic was much stronger inside the irradiated lines (Fig.1(b); the incident beam is large enough to encompass two grating lines). This enhancement could be explained by modification of either the $\chi^{(3)}$ or the frozen-in electric field.

For normally incident irradiation of a single grating line, clear second harmonic lobes were observed, one on each side (Fig.l(c)). By recording the same image in the far field, the second harmonic in these lobes was proven to be $\pi$ out of phase by the presence of a gap in the centre of the image (Fig. 1(d)). In the normally incident case, the two-lobe second harmonic distribution could be explained assuming $\chi^{(2)}=3 \chi^{(3)} E_{D C}$ and that a component of the electric field parallel to the surface of the silica glass plate is present at the interface between the irradiated and nonirradiated region (Fig.2).

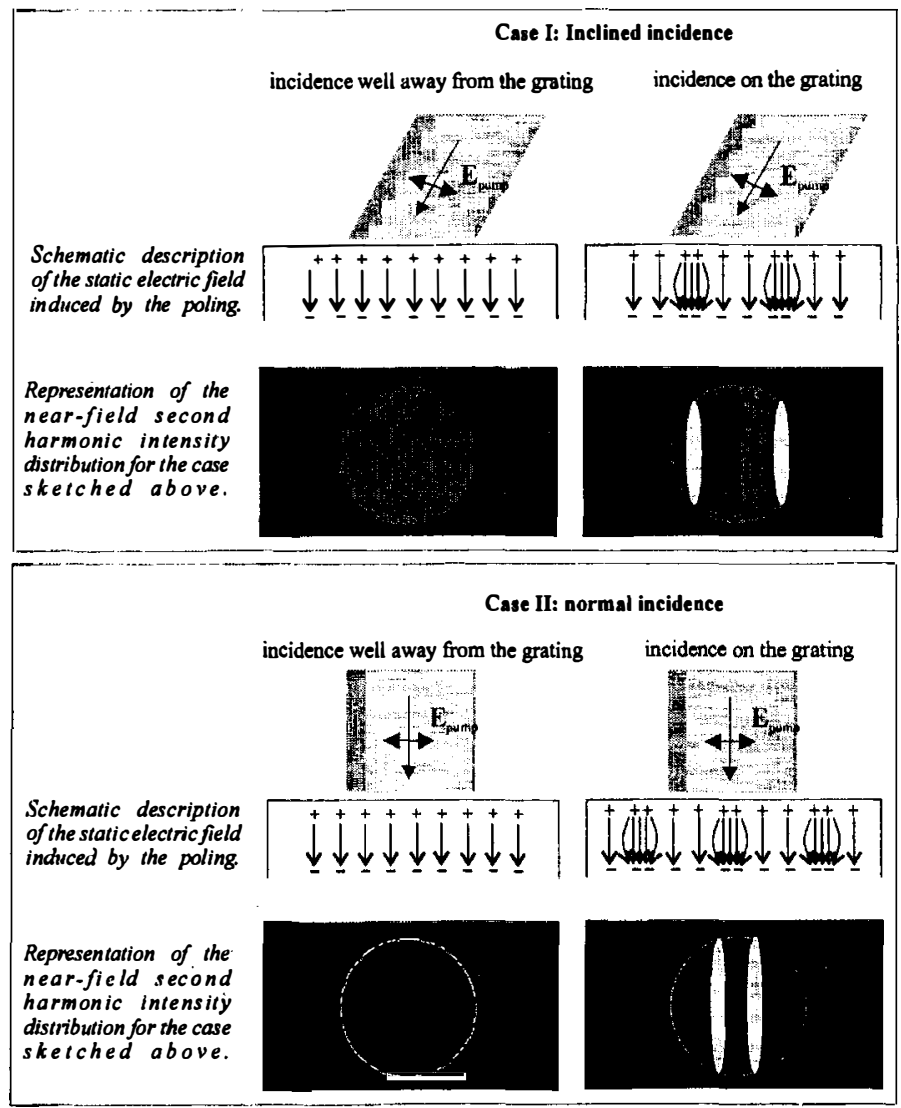

Fig. 2. A schematic description of the static electric field distribution that may explain the experimental data shown in Fig.1.

In conclusion we have demonstrated a new technique for glass poling which involves femtosecond irradiation of the sample with subsequent thermal poling. We observed enhancement of second order nonlinearity in the treated regions. The technique was used for $\chi^{(2)}$ grating fabrication. A detailed study of the glass modification responsible for the $\chi^{(2)}$ modulation is in progress.

[1] R.A. Myers, N. Mukherjee, S.R.J. Brueck "Large second-order nonlinearity in poled fused silica," Opt. Lett., 16, $1732-1734$ (1991).

[2] V. Pruneri, G. Bonfrate, P.G. Kazansky, D.J. Richardson, N.G. Broderick, J.P deSandro, C. Sommineau, P. Vidakovic, J.A. Levenson,

"Greater than $20 \%$ efficient frequency doubling of $1532 \mathrm{~nm}$ nanosecond pulses in quasi-phase-matched germanosilicate fibres," Opt. Lett.,

24, $208-210(1999)$. 3 S. Montant, H. Guillet de Chatellus, E. Freysz, "Laser -induced quasi-phase matching in thermally poled glasses," Opt. Lett., $26,837-839$

(2001) Y. Kondo, K. Nouchi, T. Mitsuyu, M. Watanaba, P.G. Kazansky, K. Hirao "Fabrication of long period gratings by focused irradiation of

infrared femtosecond laser" Opt. Lett., 24, 646-648 (1999). Misawa, J. Nishii “Generation and recombination of defects in vitreous silica induced by irradiation with a near-infrared femtosecond laser"J. Phys. Chem. B., 104, 3450-3455 (2000). 